\title{
Evaluation of dental material series from patients with dental prostheses and suspicion of delayed hypersensitivity*
}

\author{
Fernanda Cortinhas Yoshimura ${ }^{1}$ \\ Ruppert Ludwig Hahnstadt ${ }^{2}$
}

\author{
Victor do Espirito Santo Cunha² \\ Mário Cezar Pires ${ }^{1,3}$
}

DOI: http:/ /dx.doi.org/10.1590/abd1806-4841.20164116

\begin{abstract}
BACKGROUND: Patients with oral sensitivity are common in our practice. Allergic contact dermatitis is one of the most frequent etiologies.

Oвjестіves: Evaluate oral contact dermatitis using the Brazilian standard series and complementary dental series in patients using dental prostheses, with or without oral complaints. Determine specific dental Brazilian series. MeTHODS: Patients using dental prostheses with or without oral complaints realized patch tests. Brazilian standard series and complementary dental series were used according to ICDRG recommendations. The results were analysed according to age, sex, race, atopic conditions and symptoms associated.

REsULTS: From 54 patients tested, 34 (63\%) were positive at least to one substance. Nineteen had oral complaints, such as burning mouth, itch or oral erythema. There was no association between atopic condition and tests results. Without the oral series, just 23(42,6\%) patients had a positive result. Using the Brazilian standard series with the complementary dental series we improved the positivity of the patch test to $47 \%$.

CONCLUSION: In patients using prostheses and with oral complaints, patch tests with Brazilian standard series with complementary dental series improve the tests positivity.

Keywords: Dental prosthesis; Hypersensitivity; Patch tests; Prostheses and implants; Skin tests
\end{abstract}

\section{INTRODUCTION}

Frequently, patients seek dermatologic and dental clinics with oral complaints and often there is suspicion of allergic contact dermatitis (ACD). Several chemicals are commonly used in daily hygiene and oral treatments, both in restorations and in dental prostheses, and some of which are contact allergens. In Brazilian clinical practice, mercury, amalgam component, acrylates, metals and flavoring agents are examples of widely used materials. Main oral clinical complaints are burning sensation, cheilitis, stomatitis, lichenoid reactions and erythema. Allergic contact dermatitis is a delayed hypersensitivity reaction, type IV in Gell and Coombs classification, being most often triggered by haptens, e.g., incomplete antigens with low molecular weight. ${ }^{1}$ Often the etiology cannot be elucidated based only on clinical findings, requiring the performance of epicutaneous patch test, considered the gold standard for diagnosing ACD. ${ }^{1}$ Brazilian standard patch tests contains some important allergens, present in dental materials, but other substances used in dental materials are not present in this series.

Received on 15.10 .2014

Approved by the Advisory Board and accepted for publication on 23.03.2015

Study performedat Clínicas de Dermatologia e de Alergia do Hospital do Servidor Público Estadual de São Paulo - São Paulo (SP), Brazil.

Financial Support: CAPES scholarship.

Conflict of interest: Contact tests provided by FDA-Allergenic Laboratory

Instituto de Assistência Médica ao Servidor Público Estadual de São Paulo - São Paulo (SP), Brazil

Clínica privada - Rio de Janeiro (RJ), Brazil.

Complexo Hospitalar Padre Bento de Guarulhos - Guarulhos (SP), Brazil.

(C)2016 by Anais Brasileiros de Dermatologia 
The objective of this study is to establish a specific dental test series, by conducting patch tests with the Brazilian standard and a complementary dental series in patients with or without oral allergy complaint and using prostheses.

\section{METHODS}

This protocol and its informed consent were reviewed and approved by the Research Ethics Committee of the Instituto de Assistência Médica ao Servidor Público Estadual de São Paulo.

The study design was characterized as individual, analytical, longitudinal and observational. For the sample size setting, we used the Netquest software with ISO26362 certificate for the calculation. Thus, assuming 200 patch tests performed annually in the Hospital do Servidor Público Estadual de São Paulo, with 5\% margin of error, 95\% confidence level and $5 \%$ heterogeneity, the minimum sample was defined as 54 patients.

From August 2010 to March 2012, patients of the Dermatology and Allergy services of the Hospital do Servidor Público Estadual de São Paulo were selected, and the type of selection was characterized as non-probabilistic. All selected patients were using dental prostheses, with or without oral complaints.

Inclusion criteria was: use of dental prostheses, total (fully acrylic) or partially removable (acrylic and steel), with a history of at least 3 months of use, in patients of any gender and ethnicity, aged $>18$.

Patients with signs and symptoms of immune suppression; uncontrolled diabetes; pregnancy; history of use of immunosuppressive drugs in the last 30 days, including oral or depot corticosteroids; use of topical corticosteroids on the back (in the last 15 days); consumptive diseases; sun exposure on the back in the last 15 days and according to the investigator; any condition or medication that could interfere with the performance of the patch test; patients unable to understand the informed consent and individuals with mental disabilities were excluded.

All patients underwent clinical history and dermatological, oral and facial physical examination, and the data were recorded on standardized case report forms. We consider as oral allergy complaints: burning sensation; swelling; itching; labial and peri-oral erythema. History of atopic diseases such as rhinitis, asthma and atopic dermatitis was questioned.

All patients were submitted to contact tests. The test method followed the rules of International Contact Dermatitis Research Group and we used Finn Chambers $^{\circledast}$ (Smart Pratice ${ }^{\circledR}$ ) technique, applied on the backs of patients (superior line from the scapula to the waist, keeping $5 \mathrm{~cm}$ away from the spine). Readings were performed after 48 (with drawal) and 96 hours of ap- plication of the plates.

The test series used Brazilian standard (Patchkit Standard ${ }^{\circledR}$ ) and complementary dental series suggested by this study (Chart 1). Although 30 substances were tested in the Brazilian standard series, we analyzed only 11 that we considered as standard/prostheses series. Chart 1 shows the tested substances, including 11 constituents of the standard/prostheses series and 8 of the complementary dental series for a total of 19 allergens. Petrolatum was used as negative control test.

Criteria used for reading were those recommended by International Contact Dermatitis Research Group (ICDRG) and Brazilian Group of Studies on Contact Dermatitis (GBEDC):

- negative $(-)=$ no reaction;

- doubtful (?)= mild erythema presence;

- weak positive $(+)=$ well-defined erythema and papules;

- strong positive $(++)=$ erythema, papules and vesicles;

- very strong positive $(+++)=$ erythema, papules and coalescing vesicles forming blisters;

- $\mathrm{IR}=$ irritative

Tests resulting in growing were considered positive, that is, reading of 96 hours $\geq$ reading of 48 hours. Tests with more than 3 positive unrelated substances were repeated after 30 days due to the possibility of excited skin syndrome. ${ }^{2}$

Age, sex and skin color were assessed and test results with standard/prostheses series and complementary dental series regarding positivity; presence or absence of atopy and associated symptoms were also evaluated.

Results were statistically analyzed using the chisquare test and the test for two proportions, through SPSS and Biocalc programs.

\section{RESULTS}

We analyzed a total of 54 patients; mean age was 61.24 years, minimum 42 and maximum 84 years, median 62 and mode 58 years.

Regarding the time of use of the prostheses, mean time was 15.34 years, minimum 3 months and maximum 40 years, median 10 years.

The group consisted of $50(92.6 \%)$ female patients and $4(7.4 \%)$ male; regarding skin color, 38 (70.4\%) were white, followed by $10(18.5 \%)$ blacks, 5 $(9.3 \%)$ browns and $1(1.9 \%)$ yellow.

Of the 54 patients tested, $34(63 \%)$ presented positive results for at least one reagent of dental materials. Total of positive substances was 69 , which is equal to $6.72 \%$ of all tested substances. Frequency of reagents of the tests is specified in table 1. 
Снart 1: Substances tested, including the Brazilian standard series and complementary dental series

\begin{tabular}{|lll|}
\hline $\mathrm{N}^{\mathrm{o}}$ & Substance & Concentration and \\
& & vehicle \\
\hline 1 & Anthraquinone & Petrolatum2\% \\
$* 2$ & Balsam Peru & Petrolatum25\% \\
3 & PPD(MIX) & Petrolatum0,4\% \\
$* 4$ & Hydroquinone & Petrolatum1\% \\
$* 5$ & Potassium dichromate & Petrolatum0,5\% \\
6 & Propylene glycol & Petrolatum10\% \\
7 & Para- tertiary-butylphenol & Petrolatum1\% \\
8 & Neomycin & Petrolatum20\% \\
9 & Irgasan & Petrolatum1\% \\
10 & Kathon CG & Petrolatum0,5\% \\
$* 11$ & Cobalt chloride & Petrolatum1\% \\
12 & Lanolin & Petrolatum30\% \\
13 & Tiuram(MIX) & Petrolatum1\% \\
14 & Ethylenediamine & Petrolatum1\% \\
$* 15$ & Perfume (MIX) & Petrolatum7\% \\
16 & Mercapto (MIX) & Petrolatum2\% \\
$* 17$ & Benzocaine & Petrolatum5\% \\
18 & Quaternium 15 & Petrolatum0,5\% \\
19 & Quinoline(MIX) & Petrolatum6\% \\
20 & Nitrofurazone & Petrolatum1\% \\
21 & Paraben(MIX) & Petrolatum15\% \\
$* 22$ & Epoxy resin & Petrolatum1\% \\
$* 23$ & Thimerosal & Petrolatum0,05\% \\
24 & Turpentine & Petrolatum10\% \\
25 & Carba(MIX) & Petrolatum3\% \\
26 & Promethazine & Petrolatum1\% \\
$* 27$ & Nickel sulphate & Petrolatum5\% \\
$* 28$ & Colophonium & Petrolatum20\% \\
29 & Paraphenylenodiamine & Petrolatum1\% \\
$* 30$ & Formaldehyde & Water1\% \\
$* *$ & Mercury & Petrolatum0,5\% \\
$* *$ & Eugenol & Petrolatum2\% \\
$* *$ & Methyl methacrylate solid & Petrolatum2\% \\
$* *$ & Methyl methacrylate & Petrolatum2\% \\
$* *$ & Vanillin & Petrolatum10\% \\
$* *$ & Ammonium sulfate & Petrolatum2,5\% \\
$* *$ & Benzoyl peroxide & Petrolatum1\% \\
$* *$ & Vanillin (alcoholic extract) & Petrolatum10\% \\
\hline & & \\
\hline
\end{tabular}

* Substances of the Brazilian standard series used in dental materials (material kindly provided by FDA-Allergenic Laboratory).

** Complementary dental series substances.

Given the high positivity for benzoyl peroxide $1 \%$ (BP), a parallel study was conducted to evaluate the relevance of its positivity in patch tests in patients with dental prostheses and control group. For the control group, we used the same methodology of this study in 45 patients nonusers of dental prostheses and aged $\geq 42$ years. Of the 54 patients using dental prostheses, we found 17 with positive tests for BP, com-
TABLE 1: Frequency of positive substances to patch tests and frequency of oral complaints in 54 patients with dental prostheses tested from August 2010 to March 2012, in the Dermatology and Allergy Service of the Hospital do Servidor Público Estadual de São Paulo

\begin{tabular}{lll}
\hline & Positive & $\begin{array}{l}\text { Oral } \\
\text { complaints }\end{array}$ \\
& & $\mathrm{N} \%$ \\
\hline Allergens & $22.89 \%$ & $13.12 \%$ \\
Balsam Peru 25\% & $1014.49 \%$ & $618.75 \%$ \\
Thimerosal 0.05\% & $45.79 \%$ & $26.25 \%$ \\
Cobalt Chloride1\% & $22.89 \%$ & $0-$ \\
Potassium dichromate0.5\% & $1014.49 \%$ & $515.63 \%$ \\
Nickel sulfate 2.5\% & $22.89 \%$ & $26.25 \%$ \\
Colophonium20\% & $11.44 \%$ & $13.12 \%$ \\
Epoxy resin1\% & $34.34 \%$ & $26.25 \%$ \\
Perfume (Mix) 7\% & $22.89 \%$ & $0-$ \\
Formaldehyde 1\% & $22.89 \%$ & $0-$ \\
Benzocaine 5\% & $0-$ & $0-$ \\
Hydroquinone 1\% & $1724.63 \%$ & $618.75 \%$ \\
*Benzoyl peroxide 1\% & $68.69 \%$ & $39.40 \%$ \\
*Vanillin (alcoholic extract) 10\% & $34.34 \%$ & $13.12 \%$ \\
*Eugenol 2\% & $22.89 \%$ & $13.12 \%$ \\
*Mercury 0.5\% & $11.44 \%$ & $13.12 \%$ \\
*Methyl methacrylate2\% & $11.44 \%$ & $13.12 \%$ \\
*Vanillin 10\% & $11.44 \%$ & $0-$ \\
*Methyl methacrylate solid 2\% & $0-$ & $0-$ \\
*Ammonium sulfate 2.5\% & $69100 \%$ & $32100 \%$ \\
Total & & \\
& &
\end{tabular}

${ }^{*}$ Complementary dental series substances.

pared with 19 in the control group, resulting in the $p$-value $=0.27$. There was no association between the use of prostheses and positivity to benzoyl peroxide $1 \%$ in the patch test. We conclude that BP is an important allergen even in nonusers of dental prostheses, as it is frequently prescribed for common skin conditions such as acne, which could explain the high positivity observed in the control group.

Thus, we performed a study of complementary dental series with and without this reagent. Five groups of patch tests series for the analysis of positive and negative results to the reagents were formed:

- Brazilian standard/ prostheses series (11 substances);

- Complementary dental series (8 substances);

- Complementary dental series without BP (benzoyl peroxide $1 \%$ );

- Brazilian standard/ prostheses series plus complementary dental series;

- Brazilian standard/ prostheses series plus complementary dental series without BP. 
Table 2 shows the distribution of positive and negative results in the group of test series formed.

The standard/ prostheses series obtained 23 (42.6\%)positive results; with the addition of the complementary dental series the result was $34(63 \%)$, thus increasing by $47 \%$ the positivity of tests when we add the two series (Table 3).

When we evaluated the positive substances in relation with the total of tested substances $(1,026)$, the standard/ prostheses series obtained 38 positive substances; with the addition of the complementary dental series, which obtained 31 positive substances, i.e., standard/ prostheses series + complementary dental series, the result was 69 substances, presenting an increase of $81.57 \%$ in positivity (Table 4 ).
When we removed benzoyl peroxide, i.e., in the standard/ prostheses series + complementary dental series without BP, the result is 52 positive substances, increasing the positivity in $36 \%$.

Regarding atopy, 25 (46.3\%) patients were negative and 29 (53.7\%) were positive, where $22(40.74 \%)$ had rhinitis, $6(11.11 \%)$ had asthma and rhinitis, and only $1(1.85 \%)$ had asthma. No patients presented atopic dermatitis.

In table 5 we analyzed the association of the presence of atopy with the results of the tests series of the groups formed.

Regarding oral complaints considered allergic, $19(35.2 \%)$ patients were positive and $35(64.8 \%)$ were negative. One patient presented two allergic reac-

TABLE 2: Distribution of series and results in 54 patients with dental prostheses tested from August 2010 to March 2012, in the Dermatology and Allergy Service of the Hospital do Servidor Público Estadual de São Paulo

\begin{tabular}{lllllll}
\hline Series & Negative & \multicolumn{3}{c}{ Positive } & \multicolumn{2}{c}{ P-value } \\
& $\mathrm{N}$ & $\%$ & $\mathrm{~N}$ & $\%$ & \\
\hline Complementary without BP & 42 & $77.8 \%$ & 12 & $22.2 \%$ & $<0.001$ \\
Complementary & 33 & $61.1 \%$ & 21 & $38.9 \%$ & 0.021 \\
Standard/prostheses & 31 & $57.4 \%$ & 23 & $42.6 \%$ & 0.124 \\
Standard/prostheses+Complementary & 20 & $37.0 \%$ & 34 & $63.0 \%$ & 0.007 \\
Standard/prostheses+Complementary without BP & 27 & $50.0 \%$ & 27 & $50.0 \%$ & 1.000 \\
\hline
\end{tabular}

BP:Benzoyl peroxide $1 \%$

TABLE 3: Association of test results of standard/ prostheses series + complementary dental series and standard/ prostheses in 54 patients with dental prostheses tested from August 2010 and March 2012, in the Dermatology and Allergy Service of the Hospital do Servidor Público Estadual de São Paulo

\begin{tabular}{lll}
\hline Series & Standard/prostheses+ complementary & Standard/prostheses \\
\hline Positive & $34(63 \%)$ & $23(42.6 \%)$ \\
Negative & $20(37 \%)$ & $31(57.4 \%)$ \\
Total & $54(100 \%)$ & $54(100 \%)$ \\
\hline
\end{tabular}

$\mathrm{OR}=2.291 \mathrm{CI}=1.059-4.959 \mathrm{P}=0.035$

TABLE 4: Comparison of positive substances of standard/ prostheses series + complementary dental series with positive substance of the standard/ prostheses series in 54 patients with dental prostheses tested from August 2010 to March 2012, in the Dermatology and Allergy Services of the Hospital do Servidor Público Estadual de São Paulo

\begin{tabular}{llll}
\hline & standard/ protheses +complementary & standard/ protheses & Total \\
\hline$(+)$ & 69 & 38 & 107 \\
$(-)$ & 957 & 988 & 1945 \\
Total & 1026 & 1026 & 2052 \\
\hline
\end{tabular}


TABLE 5: Association of atopy with series of tests in 54 patients with dental prostheses tested from August 2010 to March 2012, in the Dermatology and Allergy Service of the Hospital do Servidor Público Estadual de São Paulo

\begin{tabular}{|c|c|c|c|c|c|c|c|c|}
\hline \multirow[t]{2}{*}{ Atopy } & & \multicolumn{2}{|c|}{ Yes } & \multicolumn{2}{|c|}{ No } & \multicolumn{2}{|c|}{ Total } & \multirow[t]{2}{*}{ P-value } \\
\hline & & $\mathbf{N}$ & $\%$ & $\mathbf{N}$ & $\%$ & $\mathbf{N}$ & $\%$ & \\
\hline \multirow[t]{2}{*}{ Complementary without BP } & Negative & 20 & $80 \%$ & 22 & $76 \%$ & 42 & $78 \%$ & 0.715 \\
\hline & Positive & 5 & $20 \%$ & 7 & $24 \%$ & 12 & $22 \%$ & \\
\hline \multirow[t]{2}{*}{ Complementary } & Negative & 14 & $56 \%$ & 19 & $66 \%$ & 33 & $61 \%$ & 0.474 \\
\hline & Positive & 11 & $44 \%$ & 10 & $34 \%$ & 21 & $39 \%$ & \\
\hline \multirow[t]{2}{*}{ Standard/prostheses } & Negative & 13 & $52 \%$ & 18 & $62 \%$ & 31 & $57 \%$ & 0.456 \\
\hline & Positive & 12 & $48 \%$ & 11 & $38 \%$ & 23 & $43 \%$ & \\
\hline Standard/prostheses+ & Negative & 8 & $32 \%$ & 12 & $41 \%$ & 20 & $37 \%$ & 0.477 \\
\hline Complementary & Positive & 17 & $68 \%$ & 17 & $59 \%$ & 34 & $63 \%$ & \\
\hline Standard/prostheses+ & Negative & 12 & $48 \%$ & 15 & $52 \%$ & 27 & $50 \%$ & 0.785 \\
\hline Complementary without BP & Positive & 13 & $52 \%$ & 14 & $48 \%$ & 27 & $50 \%$ & \\
\hline
\end{tabular}

PB: Benzoyl Peroxide

TABLE 6: Association of Oral complaints with series of tests in 54 patients with dental prostheses tested from August 2010 to March 2012, in the Dermatology and Allergy Service of the Hospital do Servidor Público Estadual de São Paulo

\begin{tabular}{lllllllll}
\hline Oral complaint & & \multicolumn{3}{c}{ Yes } & \multicolumn{2}{c}{ No } & \multicolumn{3}{c}{ Total } & \multicolumn{2}{c}{ P-value } \\
& & $\mathbf{N}$ & $\%$ & $\mathbf{N}$ & $\%$ & $\mathbf{N}$ & $\%$ & \\
\hline Complementary without BP & Negative & 29 & $83 \%$ & 13 & $68 \%$ & 42 & $78 \%$ & 0.223 \\
& Positive & 6 & $17 \%$ & 6 & $32 \%$ & 12 & $22 \%$ & \\
Complementary & Negative & 23 & $66 \%$ & 10 & $53 \%$ & 33 & $61 \%$ & 0.346 \\
& Positive & 12 & $34 \%$ & 9 & $47 \%$ & 21 & $39 \%$ & \\
Standard/prostheses & Negative & 23 & $66 \%$ & 8 & $42 \%$ & 31 & $57 \%$ & 0.094 \\
& Positive & 12 & $34 \%$ & 11 & $58 \%$ & 23 & $43 \%$ & \\
Standard/prostheses+ & Negative & 16 & $46 \%$ & 4 & $21 \%$ & 20 & $37 \%$ & 0.073 \\
Complementary & Positive & 19 & $54 \%$ & 15 & $79 \%$ & 34 & $63 \%$ & \\
Standard/prostheses+ & Negative & 21 & $60 \%$ & 6 & $32 \%$ & 27 & $50 \%$ & 0.046 \\
Complementary without BP & Positive & 14 & $40 \%$ & 13 & $68 \%$ & 27 & $50 \%$ & \\
\hline
\end{tabular}

PB: Benzoyl Peroxide

tions simultaneously. Among the positive results, oral burning was the most frequent complaint: $10(50.0 \%)$, followed by labial itching and gingival erythema: 2 $(10.0 \%)$ each; and lip burning, swelling of the mouth, facial rash, labial erythema, tongue erythema and tongue swelling: 1 (5.0\%) each.

Table 1 shows the frequency of positive substances and the frequency of oral complaints in patients with positivity for respective substances.

In patients with positivity to the substances in the standard/ prostheses series, the frequency of oral complaints was 19 (59.37\%); while in the complementary dental series was $13(40.62 \%)$. Adding the complaints of the two series the total was 32 , equivalent to an increase of $40 \%$ in oral complaints when we added the complementary dental series.

We analyzed the association of oral complaints with the results of test series of the formed groups (Table 6).
Regarding the type of prostheses used by patients, only 28 were using partially removable prostheses, 22 only total prostheses and 4 partially removable prostheses and total prostheses simultaneously. We found no association between the type of prostheses and the test results. With the addition of the removable prostheses, we found a p-value $=0.13$, and adding the total prostheses, p-value was 0.30 .

\section{DISCUSSION}

Frequently, patients present oral and perioral complaints. This region is exposed to a diverse range of materials, making it difficult to identify the cause. Oral hypersensitivity to dental materials is rare, probably due to some factors such as: the presence of saliva in the mouth, which leads to dilution and facilitates the removal of allergens; keratinization in some areas of the mucosa, hindering the union of the hapten; high local tissue vascularization, enabling the removal of al- 
lergens and mechanical resistance of the oral mucosa. ${ }^{3}$

To assist in the diagnosis and identification of allergic contact dermatitis, we used the patch test. In Brazil we have the Brazilian standard series, which has several allergens, some being found in materials used in Brazilian dentistry, particularly in users of prostheses. We selected other widely used materials in Brazilian clinic for analysis, thus establishing a specific Brazilian dental series.

Fifty-four patients were tested, with a predominance of skin color white, followed by black, brown and yellow. This distribution is in accordance with the general population treated at the Hospital do Servidor Público Estadual de São Paulo, and did not lead to differences in results. We found few studies on the prevalence of ACD in relation to skin color or race, such as the one published by Rochelle, where there is a higher incidence in whites, followed by blacks and yellows, which is in line with our results. ${ }^{4}$

Regarding gender, there was a prevalence of female patients $(92.6 \%)$, which was also observed in other studies. 5,6 Moreover, there is evidence that the incidence of ACD is similar in both sexes, and the difference is generally related to the intensity of exposure to allergens. ${ }^{7}$

The age of our sample was $42-84$ years, mean 61.24 years, median 62 years and mode 58 years. All patients were using dental prostheses, for this reason we did not test patients under age. Mean time of prostheses use was 15.34 years. Some older patients (70-84 years) used total prostheses since 20 years, resulting in a maximum time of use of 40 years. In studies performed, mentioning patients using prostheses (usually related to clinical cases), the age range was similar. ${ }^{8-10}$

Of the 54 patients tested, 34 (63\%) were positive for at least one reagent of dental materials. The total positive substances was 69 , which is equal to $6.72 \%$ of the total tested. Of the 19 substances studied, 17 had at least one positive result. Benzoyl peroxide presented the highest frequency (24.63\%), followed by nickel sulfate and thimerosal (14.49\%), vanilla extract in acetone $(8.69 \%)$, cobalt chloride $(5.79 \%)$, eugenol and perfume (mix) $(4.34 \%)$. Other substances presented between $2.89 \%$ and $1.44 \%$ of positivity in the tests. There were no positive results for ammonium sulfate and hydroquinone.

The ammonium sulfate is used in toothpastes with ammonia, considered an irritant and with little positivity in patch tests. Kanerva et al obtained a sample of 1271 patients and $2.2 \%$ of irritating results and only $0.1 \%$ are allergic to this substance. ${ }^{11}$ Torgenson et al found $1.4 \%$ positive results in 284 patients tested. ${ }^{4}$ The study sample was small to prove its relevance in the tests series, being necessary a larger number of tests.

Hydroquinone did not have positive results in our study, but this substance presents low positivity cited by other authors. ${ }^{5,11,12}$ Moreover, this is an important substance in the polymerization process of the resins, with the negative result also considered important as it can exclude it as a causative agent of allergic reaction.

As mentioned before in this paper, the positivity of benzoyl peroxide $1 \%(24.63 \%)$ was high. This is considered a very irritating substance and its allergen potential appears eventually. ${ }^{5}$ Some authors like Yoshimura et al question the value of BP as contact dermatitis agent. ${ }^{13}$ For the correct interpretation of weak positive $(+)$ to benzoyl peroxide $1 \%$, special care must be taken in the analysis of the patient's medical history and the growing aspect of the test, that is, reading of 96 hours greater than reading of 48 hours. ${ }^{14}$

We believe that patients with weak positive test for BP should conduct a repeated open application test (ROAT), as cited in the literature. ${ }^{15}$ In this test, the patient openly applies the substance in the forearm folds twice daily for 10 to 14 days; at the slightest sign of inflammation (itchiness, erythema, scaling), the use should be discontinued. The rapid improvement favors the irritation, and if the symptoms persist, it is probably an allergy. In this study, we do not encourage the realization of ROAT in those patients with positivity to $\mathrm{BP}$, because it is not our objective, but we believe that in daily practice this can be easily done. Benzoyl peroxide at higher concentrations, from $5 \%$ to $10 \%$, is widely used in the treatment of acne, commonly causing primary irritation, but it is also mentioned to cause allergies in sensitive patients. Findings of great positivity in patients nonusers of prostheses may be due to prior use of this substance to treat acne or primary irritation. All patients were asked about the use of acne products containing benzoyl peroxide, but the answers were not considered safe because patients did not remember or had doubts about it. Although the relevance of benzoyl peroxide $1 \%$ can be questioned, we decided to keep it in the dental series because it is present in most resins used in the manufacture of prostheses in Brazil (Lucitone 550 ${ }^{\circledR}$ QC-20 ${ }^{\circledR}$, VIPI Cril Plus $^{\circledR}, \mathrm{TDV}^{\circledR}$, Palaton $^{\circledR}$ ) and some authors, such as Dejobert, who presented clinical cases demonstrating that the presence of benzoyl peroxide in prosthetics was responsible for allergic contact dermatitis (ACD), also analyzed this substance. ${ }^{16}$ Being a substance frequently used in prostheses, in case of positive patch test, a more detailed research by history and performance of ROAT can prove its relevance in the diagnosis of ACD. The prostheses replacement by another without the use of BP with improvement of symptoms is the best evidence for the importance of this substance in patients with oral complaints and tested positive for BP. 
Regarding the distribution and test results of the series studied, we observed that the standard/ prostheses series resulted in $42.6 \%$ of positivity, and when we added the results of the complementary dental series $(38.9 \%)$, we reached $63 \%$ positive substances. Even if we do not consider BP positive cases, we found $50 \%$ of positivity with the tested series. Thus, when we add complementary substances suggested in this study to the standard/ prostheses series, the positivity rate of the tests increased by $47 \%$.

Evaluating the distribution of positive substances according to the tests series, we observed that adding 31 positive substances of the complementary dental series with 38 positive substances of the standard/ prostheses series (total of 69 substances), we obtained an increase of $81.57 \%$ in the positivity (Table 4 ).

Regarding the presence of atopy, 29 (53.7\%) were positive; of those, $40.74 \%$ had rhinitis, $11.11 \%$ had rhinitis and asthma, and only $1.85 \%$ had asthma, there was no patient with atopic dermatitis. At the association of test results with the presence of atopy it was found no significant statistical result. Some authors report that allergic reactions are lower in atopic patients than in non-atopic patients, others suggest that atopy condition increases the contact hypersensitivity development or that it is associated with a higher frequency of positive results in patch tests than in non-atopic patients. However the relation between atopy and late hypersensitivity to dental materials had not been analyzed until Alcayaga present a study that, just as our study, showed that atopy condition is not related to the high frequency of positive patch tests for dental materials. ${ }^{3}$

There were $19(35.2 \%)$ patients with presence of oral allergy complaint, and the most frequent complaint was burning sensation $(50.0 \%)$, followed by itchiness in the lips and gingival erythema $(10.0 \%)$, lip burning, swelling in the mouth, facial rash, labial erythema, tongue erythema, and tongue swelling (5.0\%). The high frequency of burning sensation is often cited in the studies. Burning and pain sensation in the oral cavity without mucosal lesions define the Burning Mouth Syndrome. ${ }^{17,18}$ The Burning Mouth Syndrome affects elderly women, which is in accordance with the composition of our sample. Moreover, a detailed study to define the presence of this syndrome in these patients was not performed.

In the association of oral complaints with the test results, we observed a statistical association with higher relevance with the standard/ prostheses series + complementary dental series without BP $(p=0.046)$. Thus, among people without oral complaints, $60 \%$ were negative in this series. Among people with oral complaints, $68 \%$ presented positive results. When we added benzoyl peroxide $1 \%$, we increased the association between test positivity and presence of allergic complaints to $79 \%$ and the p-value was 0.073 .

When we observed the positive substances with presence of oral complaint, there is an increase of $40 \%$ of complaints with the addition of the complementary dental series to the standard/ prostheses series. Interpretation of positive substances as relevant is a challenge: changing the type of restorative material, stopping the use of prostheses for a period of time or even exchanging the prostheses components, would be the best ways to confirm whether the cause of the symptoms originates from the materials used. This conduct could not be held in our study due to the high cost, limited mobility of patients to return to consultations and lack of cooperation in relation to stop using the prostheses for a certain period. Several studies mention changing of types of materials, whether or not interrupting the clinical symptoms. ${ }^{19-21}$

We found no association between the type of prostheses (removable or total) with positivity of tests. What distinguishes one from the other is the metal portion present in the removable prostheses, because both have an acrylic piece. Many patients with total prostheses tested positive for metals; this positivity can be attributed to other components such as jewelry, or even some existing metal restorations in the mouth. There were no studies mentioning this association, but clinical case studies confirms the presence of allergens in prostheses. ${ }^{8,9}$

To determine the dental tests series suggested, we decided to delete the sulphate of ammonia and keep benzoyl peroxide $1 \%$.

\section{CONCLUSION}

In patients using dental prostheses, patch test with dental substances present in the Brazilian standard series with the addition of the complementary dental series is an important aid in the diagnosis of patients with oral complaints and use of prostheses. The specific dental series suggested by this study consists of 18 substances (specified in Table 1), as there was exclusion of the ammonium sulfate $2.5 \%$.

\section{Acknowledgements:}

Prof. Dr. José Alexandre Sittart, Prof. Dr. João Ferreira de Melo, Dr. Wilson Tartucce Aun, Dra. Maria de Fátima Rodrigues Maestre, Dra. Maria Elisa Bertocco Andrade, Wanira Márcia Maranhão, Maria Giorlando 


\section{REFERENCES}

1. Rietschel R. L., Fowler J. F. Jr. The pathogenesis of allergic contact hypersensitivity. In: Rietschel R. L., Fowler J. F. Jr editors. Fisher`s Contact Dermatitis. Philadelphia Lippincott Williams \& Wilkins; 2001.p.1-7.

2. Duarte I. Síndrome da pele excitada: revisão da literatura. An Bras Dermatol. 1995;70:153-62.

3. Rojas-Alcayaga G, Carrasco-Labra A, Danús P, Guzmán MA, Morales-Bozo I, Urzúa $B$, et al. Determination of susceptibility to sensitization to dental materials in atopic and non-atopic patients. Med Oral Patol Oral Cir Bucal. 2012;17:e320-4.

4. Torgerson RR, Davis MD, Bruce AJ, Farmer SA, Rogers RS 3rd.Contactallergy in oral disease. J Am Acad Dermatol. 2007;57:315-21.

5. Gebhart M, Geier J. Evaluation of patch test results with denture material series. Contact Dermatitis. 1996;34:191-5.

6. Hosoki M, Bando E, Asaoka K, Takeuchi H, Nishigawa K.Assessment of allergic hypersensitivity to dental materials. Biomed Mater Eng. 2009;19:53-61.

7. Duarte I, Lazzarini R, Buense R, Pires MC. Contactdermatitis. An Bras Dermatol. 2000; 75:529-48

8. Koutis D, Freeman S. Allergic contact stomatitis caused by acrylic monomer in a denture. Australas J Dermatol. 2001;42:203-6.

9. Kanerva L, Tarvainen K, Jolanki R, Estlander T. Successful coating of an allergenic acrylate-based dental prosthesis. Am J Contact Dermat. 1995;6:24-7.

10. Vozmediano J, Manrique A. Active sensitization to (meth)acrylates. Contact Dermatitis. 1998;39:314

11. Kanerva L, Rantanen T, Aalto-Korte K, Estlander T, Hannuksela M, Harvima RJ, et al. A multicenter study of patch test reactions with dental screening series. Am J Contact Dermat. 2001;12:83-7.

12. Shmidt E, Farmer SA, Davis MD. Patch-Testing with Plastic and Glues Series Allergens. Dermatitis. 2010;21:269-74.

13. Pires MC, Andrade MLB, Pires RMG, Castello RG, Yoshimura FC. Avaliação da sensibilidade ao peróxido de benzoíla e sua relevância em pacientes submetidos ao teste de contato. Rev Bras Alerg Imunopatol. 2012;35:140.

14. Ockenfels HM, Uter W, Lessmann H, Schnuch A, Geier J. Patch testing with benzoyl peroxide: reaction profile and interpretation of positive patch test reactions. Contact Dermatitis. 2009;61:209-16

15. Rietschel RL, Fowler JF Jr. Practical Aspects of Patch Testing. In: Rietschel $\mathrm{RL}$, Fowler JF Jr, editors. Fisher`s Contact Dermatitis. Philadelphia: Lippincott Williams \& Wilkins; 2001.p.10.

16. Dejobert $\mathrm{Y}$, Piette F, Thomas P. Contact dermatitis from benzoyl peroxide in dental prostheses. Contact Dermatitis. 2002;46:177-8.

17. Marino R, Capaccio P, Pignataro L, Spadari F. Burning mouth syndrome: the role of contact hypersensitivity. Oral Dis. 2009;15:255-8.

18. Schuster C, Mofarrah R, Aberer W, Kränke B. Pitfalls of patch testing with dental materials. Br J Dermatol. 2012;166:674-5

19. Gomez-dela Fuente E, Vicente-Martin FJ, Sánchez-Gilo A, López-Estebaranz JL. Reacciónliquenoide oral erosiva secundaria a sensibilización a cobalto em próteses dental. Med Cutan Iber Lat Am. 2012;40:72.

20. Raap U, Stiesch M, Reh H, Kapp A, Werfel T. Investigation of contact allergy to dental metals in 206 patients. Contact Dermatitis. 2009:60:339-43.

21. Shah M, Lewis FM, Gawkrodger DJ. Contact Allergy in Patients with Oral Symptoms: A Study of 47 Patients. Am J ContactDermat. 1996;7:146-51.
MAILING ADDRESS:

Fernanda Cortinhas Yoshimura

Rua Pedro de Toledo,1800 - Moema

04029-000 - São Paulo - SP

Brazil

Email:fe.yoshi@uol.com.br

How to cite this article: Yoshimura FC, Cunha VES, Hahnstadt RL, Pires MC. Evaluation of dental material series from patients with dental prostheses and suspicion of delayed hypersensitivity. An Bras Dermatol. 2016;91(2):141-8. 